\title{
A 3-quasiparticle isomer in neutron-rich ${ }^{183} \mathrm{Ta}$
}

\author{
T. Shizuma ${ }^{1, a}$, T. Ishii ${ }^{2,3}$, H. Makii ${ }^{2}$, T. Hayakawa ${ }^{1}$, and M. Matsuda ${ }^{3}$ \\ 1 Kansai Photon Science Institute, Japan Atomic Energy Agency, Kizugawa, Kyoto 619-0215, Japan \\ 2 Advanced Science Research Center, Japan Atomic Energy Agency, Tokai, Ibaraki 319-1195, Japan \\ 3 Department of Research Reactor and Tandem Accelerator, Japan Atomic Energy Agency, Tokai, Ibaraki 319-1195, Japan
}

Received: 24 August 2008 / Revised: 18 November 2008

Published online: 10 February 2009 - (C) Società Italiana di Fisica / Springer-Verlag 2009

Communicated by S. Kubono

\begin{abstract}
Excited states in neutron-rich ${ }^{183} \mathrm{Ta}$ have been studied using a two-neutron transfer reaction of ${ }^{181} \mathrm{Ta}\left({ }^{18} \mathrm{O},{ }^{16} \mathrm{O}\right)$. In-beam $\gamma$-rays were measured in coincidence with scattered ions detected by a highresolution $\Delta E-E$ Si telescope for reaction channel selection. Previously known 1-quasiparticle bands are extended to higher spins, and several levels including a $T_{1 / 2}=0.9(3) \mu$ s 3 -quasiparticle isomer are identified.
\end{abstract}

PACS. 21.10.Tg Lifetimes widths - 23.20.Lv $\gamma$ transitions and level energies -25.70. Hi Transfer reactions $-27.70 .+\mathrm{q} 150 \leq A \leq 189$

Information on intrinsic and rotational states of medium to high spins in neutron-rich nuclei has been limited because of the difficulty to access to such nuclei using standard fusion-evaporation reactions. Recent progress, however, in in-beam $\gamma$-ray spectroscopic techniques using deep inelastic [1-3] and multi-nucleon transfer reactions $[4,5]$ has enable us to study the yrast structure of neutron-rich nuclei. For example, high-spin states in neutron-rich ${ }^{182} \mathrm{Hf}$ have been successfully identified through a deep inelastic reaction with a ${ }^{136} \mathrm{Xe}$ beam incident on ${ }^{180} \mathrm{Hf}[1]$. Using ${ }^{18} \mathrm{O}$-induced multi-nucleon transfer reactions such as $\left({ }^{18} \mathrm{O},{ }^{16} \mathrm{O}\right),\left({ }^{18} \mathrm{O},{ }^{17} \mathrm{O}\right),\left({ }^{18} \mathrm{O},{ }^{19} \mathrm{~F}\right)$, and $\left({ }^{18} \mathrm{O},{ }^{20} \mathrm{Ne}\right)$, modest spin states of neutron-rich nuclei have been studied [4-9]. In the present work, excited states of the neutron-rich ${ }^{183} \mathrm{Ta}$ have been populated following a two-neutron transfer reaction of ${ }^{181} \mathrm{Ta}\left({ }^{18} \mathrm{O},{ }^{16} \mathrm{O}\right)$.

The experiment was carried out at the Tokai tandem accelerator facility [10] of Japan Atomic Energy Agency. The $180 \mathrm{MeV}{ }^{18} \mathrm{O}$ beam was incident on a natural Ta self-supporting foil with a thickness of $3.9 \mathrm{mg} / \mathrm{cm}^{2}$, which is thick enough to stop target-like nuclei inside the target material. Outgoing ions were detected by four sets of surface barrier Si $\Delta E-E$ detectors with a diameter of $20 \mathrm{~mm}$. These detectors were placed at $28^{\circ}$ with respect to the beam direction. Emitted $\gamma$-rays were measured with eight HP-Ge detectors, in coincidence with outgoing ions. Four of these detectors were arranged symmetrically in a plane perpendicular to the beam axis, providing $\gamma$-ray anisotropy information for the determination of transi-

\footnotetext{
a e-mail: shizuma.toshiyuki@jaea.go.jp
}

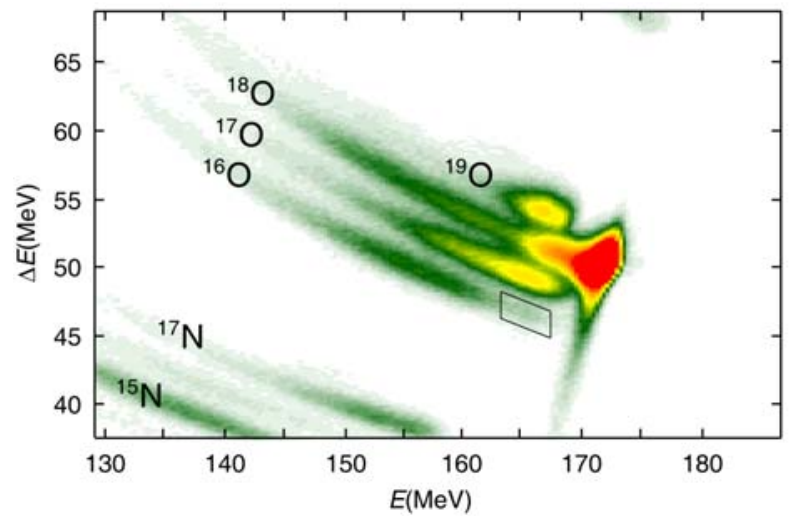

Fig. 1. The $E-\Delta E$ plot for outgoing ions measured by the $\mathrm{Si}$ detectors. The enclosed areas represent the gate window with the kinematic energies of $164-168 \mathrm{MeV}$.

tion multipole orders [4]. Energy and efficiency calibrations of the Ge detectors were made by using ${ }^{133} \mathrm{Ba}$ and ${ }^{152} \mathrm{Eu}$ standard $\gamma$-ray sources. The time difference between signals from the $\mathrm{Si}$ and Ge detectors was measured by time-to-amplitude converters (TAC). The energy and time information on outgoing ions and $\gamma$-rays were recorded event by event on magnetic tapes. A total of $2.3 \times 10^{8}$ and $6.8 \times 10^{7}$ events for particle- $\gamma$ and particle- $\gamma-\gamma$ coincidences, respectively, were collected. The details of the experimental setup are described in ref. [6].

The $E-\Delta E$ plot for outgoing ions is shown in fig. 1 . Each ions are clearly separated according to the mass 


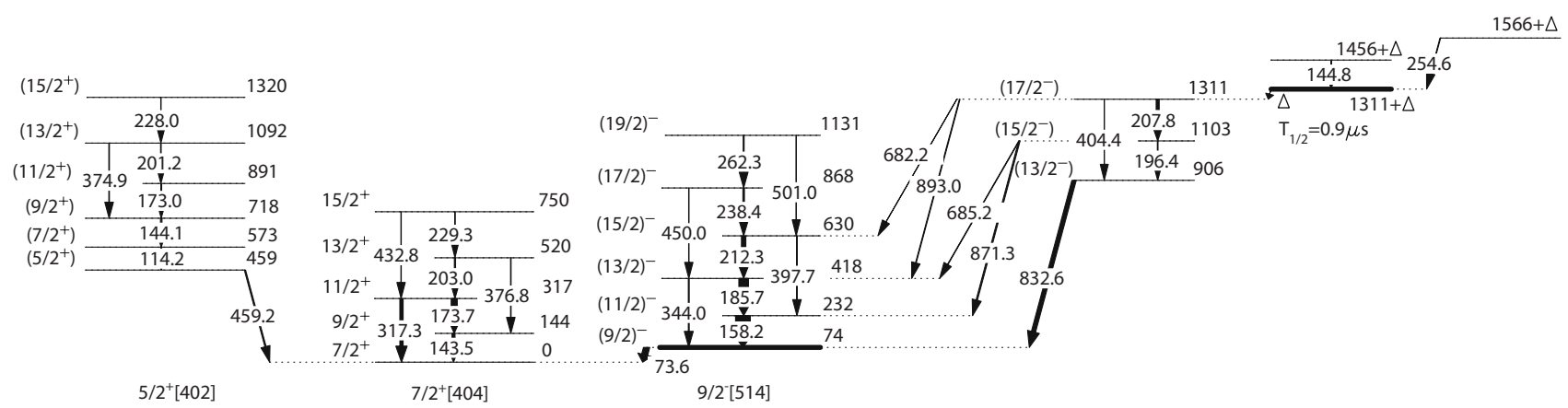

Fig. 2. The proposed level scheme for ${ }^{183}$ Ta. The Nilsson configurations are shown for each 1-quasiparticle bands. The $\gamma$-ray and level energies are given in units of $\mathrm{keV}$.

and atomic numbers. The particle energies were calibrated assuming that the most intense peak in the $E-\Delta E$ matrix corresponds to elastically scattered events of ${ }^{18} \mathrm{O}$ ions entering at the center of the each $\mathrm{Si}$ detector. In the present data analysis, the narrow gate window on ${ }^{16} \mathrm{O}$ ions, shown with the enclosed area in fig. 1 , is used to eliminate events associated with ${ }^{181} \mathrm{Ta}$ and ${ }^{182} \mathrm{Ta}$ nuclei. These can be produced by two- and one-neutron evaporation from ${ }^{183} \mathrm{Ta}$ when the compound-like ${ }^{183} \mathrm{Ta}$ is excited above the neutron separation energies. The higher-energy ${ }^{16} \mathrm{O}$ gate therefore gives clean $\gamma$-ray spectra for ${ }^{183} \mathrm{Ta}$.

The level scheme of ${ }^{183} \mathrm{Ta}$ deduced from the present experimental data is shown in fig. 2 . The ground state is assigned to be the $7 / 2^{+}$[404] 1-quasiparticle configuration, the same as other Ta isotopes with $175 \leq A \leq 185$ [11]. Levels at $74,144,459$, and $573 \mathrm{keV}$, identified in the $\beta$-decay study of ${ }^{183} \mathrm{Hf}[12]$, are confirmed. While the $144 \mathrm{keV}$ level is known as a member of the $7 / 2^{+}[404]$ band, the 459 and $573 \mathrm{keV}$ levels are based on the $5 / 2^{+}[402]$ configuration. The present data extend the $7 / 2^{+}[404]$ and $5 / 2^{+}[402]$ bands to higher spins. It is noted that the inband transition energies of these bands, which are pseudospin partners [13], are very close to each other. The $74 \mathrm{keV}$ state is based on the $9 / 2^{-}$[514] configuration, but its rotational band has not been observed. The energy spectrum of $\gamma$-rays in coincidence with the $74 \mathrm{keV}$ transition which depopulates the bandhead is shown in fig. 3(a). Rotational band members up to $I^{\pi}=\left(19 / 2^{-}\right)$are identified in this band. Analysis of the in-band $\gamma$-ray branching ratios using the rotational model expressions [14] gives an average $g_{K}$ value of $1.4(4)$ with $Q_{0}=6 \mathrm{eb}$ and $g_{R}=0.3$. This is consistent with the expected value of $g_{K}=1.29$ for the $9 / 2^{-}[514]$ configuration. Spin assignments for the observed levels are based on $\gamma$-ray anisotropy data if available. The $\gamma$-ray anisotropy is given as an intensity ratio of $\gamma$-rays detected by the Ge detectors placed in and out of the reaction plane. The ratio depends on the transition multipole order, the degree of polarization, and the mixing ratio. In practice, the intensity ratio $R\left[=I_{\gamma}(\right.$ in $) / I_{\gamma}$ (out) $]$ is greater than unity for stretched quadrupole $(\Delta I=2)$ and $R \approx 0.5$ for stretched pure dipole transitions [15]. For mixed $\Delta I=1$ transitions, the ratio varies with the mixing ratio, and e.g., $R \approx 1$ for positive small mixing ratios [15] which can be applied for the transitions of 1quasiparticle bands observed in ${ }^{183} \mathrm{Ta}$. Note that the sign

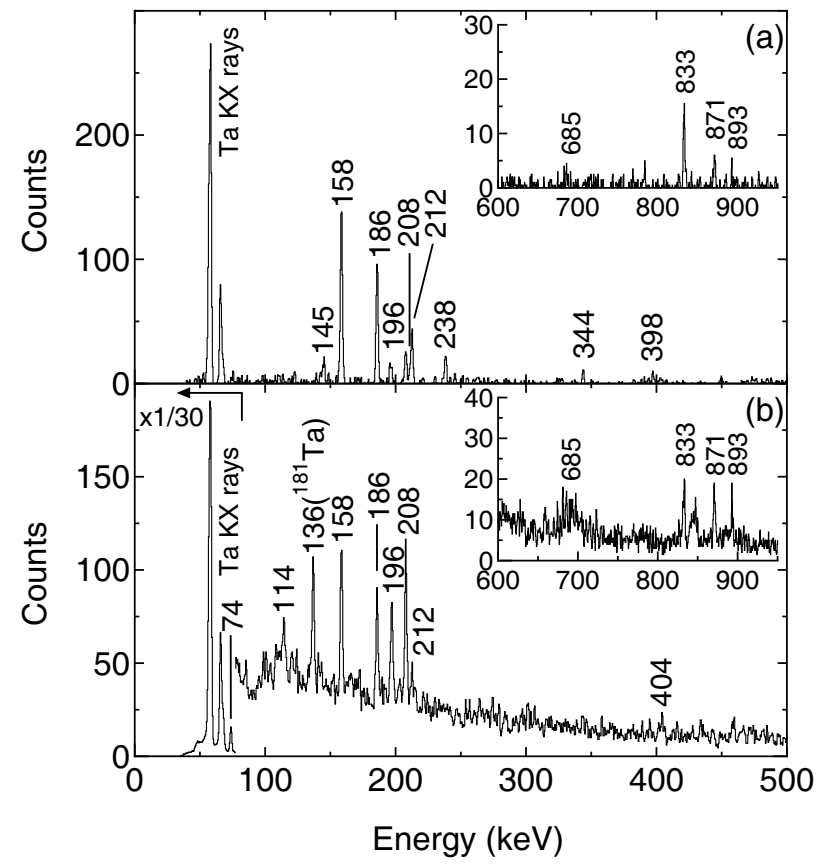

Fig. 3. The spectrum of $\gamma$-rays in coincidence with the $74 \mathrm{keV}$ transition within $2 \mu$ s correlation time is shown in panel (a). The $\gamma$-ray peaks are labeled in units of $\mathrm{keV}$. The delayed $\gamma$-ray spectrum gated on ${ }^{16} \mathrm{O}$ ions is shown in panel (b).

of the mixing ratio equals that of $\left(g_{K}-g_{R}\right) / Q_{0}$, and positive mixing rations are expected for the $\Delta I=1$ transitions in the 1-quasiparticle bands with calculated $g_{K}$ values of $1.57\left(5 / 2^{+}[402]\right), 0.63\left(7 / 2^{+}[404]\right)$, and $1.29\left(9 / 2^{-}[514]\right)$. In the spin and parity assignment, multipolarities of $M 1$ and E2 have been considered for fast in-band $\gamma$-ray transitions. Rotational alignments [16] plotted in fig. 4 for the 1-quasiparticle bands in ${ }^{183} \mathrm{Ta}$ show small values i.e., $\sim 0 \hbar$ for the $5 / 2^{+}[402]$ and $7 / 2^{+}[404]$ bands, and $\sim 0.5 \hbar$ for the $9 / 2^{-}[514]$ band, which agree with the interpretation that these bands are strongly coupled bands.

Several $\gamma$-ray transitions feeding the $9 / 2^{-}[514]$ band from levels at 906,1103 , and $1311 \mathrm{keV}$, which are assigned as $I^{\pi}=\left(13 / 2^{-}\right),\left(15 / 2^{-}\right)$, and $\left(17 / 2^{-}\right)$, respectively, from the analysis of $\gamma$-ray anisotropy data, are observed to form a rotational band structure. With the $K=13 / 2$ assignment for this band, the bandhead at 


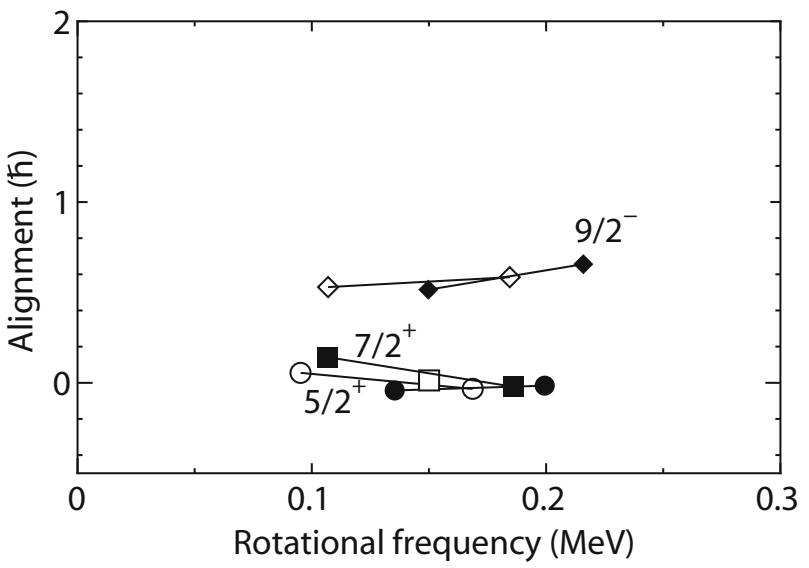

Fig. 4. Rotational alignments for the bands observed in ${ }^{183} \mathrm{Ta}$ are plotted as a function of the rotational frequency with Harris parameters $\mathfrak{I}_{0}=31.5 \mathrm{MeV}^{-1} \cdot \hbar^{2}$ and $\mathfrak{I}_{1}=32 \mathrm{MeV}^{-3} \cdot \hbar^{4}$ taken from ref. [17].

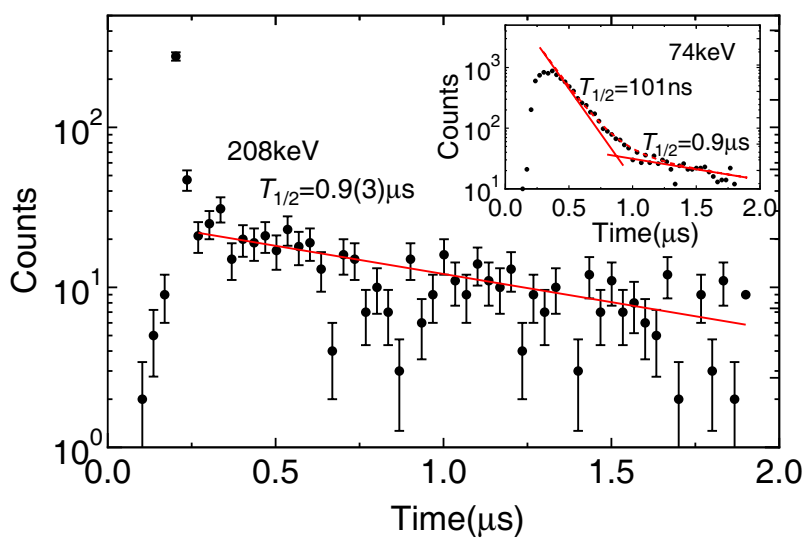

Fig. 5. The particle- $\gamma$ time difference spectrum for the $208 \mathrm{keV}$ transition is plotted. The time spectrum for the $74 \mathrm{keV}$ transition is also shown in the inset panel. Delayed components with $T_{1 / 2}=0.9 \mu \mathrm{s}$ in the $74 \mathrm{keV}$ time spectrum are associated with the decay of the newly observed isomer.

$906 \mathrm{keV}$ is too low to be a 3 -quasiparticle state, and could be a $\gamma$ vibrational state coupled to the $9 / 2^{-}[514]$ configuration which is implied by the observed inter-band transitions to the $9 / 2^{-}[514]$ band. States formed by $\gamma$ vibrations on the $9 / 2^{-}[514]$ configuration are known to exist in ${ }^{177} \mathrm{Lu}$ at $1306 \mathrm{keV}$ [18], ${ }^{177} \mathrm{Ta}$ at $899 \mathrm{keV}$ [19], and ${ }^{187} \mathrm{Re}$ at $793 \mathrm{keV}$ [20]. Information on the $\gamma$-ray energies, intensities and in- and out-of-plane intensity ratios if obtained is summarized in table 1.

From the analysis of the delayed coincidence spectrum shown in fig. 3(b), a new isomer has been identified above the $1311 \mathrm{keV}$ level. The half-life of this isomer is determined as $T_{1 / 2}=0.9(3) \mu$ s from the particle- $\gamma$ time difference spectrum shown in fig. 5. In the decay curve of the $208 \mathrm{keV} \gamma$-ray, a prompt decay component can be seen. This indicates that the $1311 \mathrm{keV}$ level depopulated by the $208 \mathrm{keV}$ transition is not isomeric itself, but it is fed from the isomer. Note that a half-life for the $74 \mathrm{keV}$ state is deduced as $T_{1 / 2}=101(20)$ ns (see fig. 5) which agrees with
Table 1. Energies $E_{\gamma}$, relative intensities $I_{\gamma}$, and initial level energies $E_{i}$ for the $\gamma$-ray transitions observed in ${ }^{183} \mathrm{Ta}$. Intensity ratios $I_{\gamma}(\mathrm{in}) / I_{\gamma}$ (out) for in-plane to out-of-plane anisotropies are also given.

\begin{tabular}{|c|c|c|c|}
\hline $\begin{array}{c}E_{\gamma} \\
(\mathrm{keV})\end{array}$ & $I_{\gamma}$ & $\begin{array}{c}E_{i} \\
(\mathrm{keV})\end{array}$ & $I_{\gamma}($ in $) / I_{\gamma}($ out $)$ \\
\hline $73.6(1)$ & $41(4)$ & 74 & $1.0(1)$ \\
\hline $114.2(1)$ & $6(2)$ & 573 & $1.1(1)$ \\
\hline $143.6(1)$ & $21(4)$ & 144 & $1.1(1)^{\mathrm{a}}$ \\
\hline 144.1(1) & $10(2)$ & 718 & $1.1(1)^{\mathrm{a}}$ \\
\hline $144.8(1)$ & $\sim 2$ & 1506 & $1.1(1)^{\mathrm{a}}$ \\
\hline $158.3(1)$ & $100(6)$ & 232 & $1.0(1)$ \\
\hline $173.0(2)$ & $6(2)$ & 891 & $1.2(1)^{\mathrm{a}}$ \\
\hline $173.7(1)$ & $37(4)$ & 317 & $1.2(1)^{\mathrm{a}}$ \\
\hline $185.7(1)$ & $65(4)$ & 418 & $1.1(1)$ \\
\hline $196.4(1)$ & $5(2)$ & 1103 & $0.8(2)$ \\
\hline $201.2(2)$ & $10(2)$ & 1092 & $1.0(3)^{\mathrm{a}}$ \\
\hline $203.0(1)$ & $6(2)$ & 520 & $1.0(3)^{\mathrm{a}}$ \\
\hline $207.8(1)$ & $21(2)$ & 1311 & $1.0(2)$ \\
\hline $212.3(1)$ & $30(3)$ & 630 & $1.0(1)$ \\
\hline $228.0(3)$ & $\sim 2$ & 1320 & $1.1(3)^{\mathrm{a}}$ \\
\hline $229.3(6)$ & $4(1)$ & 750 & $1.1(3)^{\mathrm{a}}$ \\
\hline $238.4(1)$ & $10(2)$ & 868 & \\
\hline $254.6(10)$ & $4(2)$ & 1616 & \\
\hline $262.3(1)$ & $6(2)$ & 1131 & \\
\hline $317.3(1)$ & $22(11)$ & 317 & $1.5(1)$ \\
\hline $344.0(3)$ & $6(2)$ & 418 & $1.4(2)$ \\
\hline $374.9(3)$ & $4(2)$ & 1092 & $1.9(9)^{\mathrm{a}}$ \\
\hline $376.8(10)$ & $5(3)$ & 520 & $1.9(9)^{\mathrm{a}}$ \\
\hline $397.7(3)$ & $4(2)$ & 630 & $1.5(2)$ \\
\hline $404.4(10)$ & $<1$ & 1311 & \\
\hline $432.8(6)$ & $6(2)$ & 750 & $2.2(6)$ \\
\hline $450.0(5)$ & $2(1)$ & 868 & $2.8(7)$ \\
\hline $459.2(1)$ & $8(4)$ & 459 & \\
\hline $501.0(9)$ & $2(1)$ & 1131 & $2.6(7)$ \\
\hline $682.2(10)$ & $<1$ & 1311 & \\
\hline $685.2(3)$ & $3(2)$ & 1103 & \\
\hline $832.6(1)$ & $29(5)$ & 906 & $1.3(2)$ \\
\hline $871.3(2)$ & $9(3)$ & 1103 & $1.6(2)$ \\
\hline $893.0(10)$ & $5(2)$ & 1311 & $1.7(3)$ \\
\hline
\end{tabular}

a Doublet transitions.

the adopted value of $107(11)$ ns [11] within the quoted uncertainties. The $\gamma$-ray linking the $1311 \mathrm{keV}$ state and the isomer, labeled by " $\Delta$ " in fig. 2 , has not been observed. On the basis of detection efficiency and conversion coefficient consideration, the possible energy $\Delta$ of the missing transition is deduced to be less than $50 \mathrm{keV}$ for $E 1$ and $100 \mathrm{keV}$ for $M 1$ and $E 2$. Consequently, spins and parities of $19 / 2^{ \pm}$or $21 / 2^{-}$are likely for the isomeric state. Most of these assignments, however, can be excluded by the following discussion of the hindrance factors $f_{\nu}$ per degree of $K$ forbiddenness for the isomeric transition. The $f_{\nu}$ factor is defined as $f_{\nu}=\left(T_{1 / 2}^{\gamma} / T^{W}\right)^{1 / \nu}$ where $T_{1 / 2}^{\gamma}$ and $T^{W}$ are 
the partial $\gamma$-ray half-life and the corresponding Weisskopf single-particle estimate, respectively, and $\nu$ is the order of $K$ forbiddenness, defined as $\nu=\Delta K-\lambda$ for transitions of multipole order $\lambda$. The consideration of the hindrance factor estimated for the isomeric transition can exclude the $19 / 2^{-}$assignment because of the large hindrance deduced for the isomeric transition which would be $M 1$ with $f_{\nu}=474$ assuming $\Delta=100 \mathrm{keV}$ and the $21 / 2^{-}$assignment because of the abnormally strong $E 2$ transition with $f_{\nu}=2$ assuming $\Delta=100 \mathrm{keV}$. This leads only the $19 / 2^{+}$ assignment with $f_{\nu}$ factor of $28(\Delta=50 \mathrm{keV}$ assumed $)$ which is consistent with values obtained for $K$ forbidden $E 1$ transitions in ${ }^{175} \mathrm{Ta}$ [21] and ${ }^{177} \mathrm{Ta}$ [19]. Note that the $T^{W}$ value for the $E 1$ transition was multiplied by $10^{3}$ before calculating $f_{\nu}$. From the consideration of low-lying single-particle states a $K^{\pi}=19 / 2^{+}, 3$-quasiparticle configuration of $\pi\left\{9 / 2^{-}[514]\right\} \otimes \nu 5^{-}\left\{1 / 2^{-}[510] 11 / 2^{+}[615]\right\}$ is likely assigned to the isomer. The $\nu 5^{-}$configuration is obtained by a favored spin-spin coupling associated with the empirical Gallagher-Moszkowski interaction [22] and are known in ${ }^{184} \mathrm{~W}\left(E_{x}=1285 \mathrm{keV}\right)[23]$ and ${ }^{186} \mathrm{Os}$ $\left(E_{x}=1629 \mathrm{keV}\right)$ [24]. A similar isomer with the same configuration is known at $1682 \mathrm{keV}$ in ${ }^{187} \mathrm{Re}[20]$.

In summary, excited states of neutron-rich ${ }^{183} \mathrm{Ta}$ have been populated by an ${ }^{18} \mathrm{O}$-induced two-neutron transfer reaction. Previously known one-quasiparticle bands are extended to higher spins. Several new levels, including a $T_{1 / 2}=0.9 \mu \mathrm{s}$ isomer, have been found. On the basis of the $K$ hindrance consideration, the spin and parity of $19 / 2^{+}$are deduced and a 3-quasiparticle configuration of $\pi\left\{9 / 2^{-}[514]\right\} \otimes \nu 5^{-}\left\{1 / 2^{-}[510] 11 / 2^{+}[615]\right\}$ is given for the isomer.

We thank the staff of the JAEA tandem accelerator facility for providing the ${ }^{18} \mathrm{O}$ beam.

\section{References}

1. E. Ngijoi-Yogo, S.K. Tandel, G. Mukherjee, I. Shestakova, P. Chowdhury, C.Y. Wu, D. Cline, A.B. Hayes, R. Teng, R.M. Clark, P. Fallon, A.O. Macchiavelli, K. Vetter, F.G. Kondev, S. Langdown, P.M. Walker, C. Wheldon, D.M. Cullen, Phys. Rev. C 75, 034305 (2007).

2. C. Wheldon, J. Garcés Narro, C.J. Pearson, P.H. Regan, Zs. Podolyák, D.D. Warner, P. Fallon, A.O. Macchiavelli, M. Cromaz, Phys. Rev. C 63, 011304(R) (2000).

3. T. Shizuma, T. Hayakawa, S. Mitarai, T. Morikawa, T. Ishii, Phys. Rev. C 71, 067301 (2005).

4. T. Ishii, S. Shigematsu, M. Asai, A. Makishima, M. Matsuda, J. Kaneko, I. Hossain, S. Ichikawa, T. Kohno, M. Ogawa, Phys. Rev. C 72, 021301(R) (2005).
5. T. Ishii, S. Shigematsu, H. Makii, M. Asai, K. Tsukada, A. Toyoshima, M. Matsuda, A. Makishima, T. Shizuma, J. Kaneko, I. Hossain, H. Toume, M. Ohara, S. Ichikawa, T. Kohno, M. Ogawa, J. Phys. Soc. Jpn. 75, 043201 (2006).

6. T. Shizuma, T. Ishii, H. Makii, T. Hayakawa, S. Shigematsu, M. Matsuda, E. Ideguchi, Y. Zheng, M. Liu, T. Morikawa, P.M. Walker, M. Oi, Eur. Phys. J. A 30, 391 (2006).

7. T. Shizuma, T. Ishii, H. Makii, T. Hayakawa, S. Shigematsu, M. Matsuda, E. Ideguchi, Y. Zheng, M. Liu, T. Morikawa, Eur. Phys. J. A 34, 1 (2007).

8. T. Ishii, H. Makii, M. Asai, H. Koura, S. Shigematsu, K. Tsukada, A. Toyoshima, M. Matsuda, A. Makishima, J. Kaneko, H. Toume, I. Hossain, T. Shizuma, S. Ichikawa, T. Kohno, M. Ogawa, Phys. Rev. C 76, 011303(R) (2007).

9. T. Shizuma, T. Ishii, H. Makii, T. Hayakawa, M. Matsuda, S. Shigematsu, E. Ideguchi, Y. Zheng, M. Liu, T. Morikawa, M. Oi, Phys. Rev. C 77, 047303 (2008).

10. S. Takeuchi, T. Ishii, M. Matsuda, Y. Zhang, T. Yoshida, Nucl. Instrum. Methods Phys. Res. A 382, 153 (1996).

11. ENSDF, NNDC Online Data Service, ENSDF database, http://www.nndc.bnl.gov/nndc/ensdf/.

12. L.D. McIsaac, R.G. Helmer, C.W. Reich, Nucl. Phys. A 132, 28 (1969).

13. A. Arima, M. Harvey, K. Shimizu, Phys. Lett. B 30, 517 (1969).

14. A. Bohr, B.R. Mottelson, Nuclear Structure, Vol. 2 (Benjamin, Reading, 1975).

15. S. de Groot, H. Tolhoek, W. Huiskamp, in Alpha-, Beta-, and Gamma-Ray Spectroscopy, edited by K. Siegbahn, Vol. 2 (North-Holland, Amsterdam, 1965) pp. 1199-1261.

16. R. Bengtsson, S. Frauendorf, Nucl. Phys. A 327, 139 (1979).

17. T.R. Saitoh, N. Hashimoto, G. Sletten, R.A. Bark, S. Törmänen, M. Bergström, K. Furuno, K. Furutaka, G.B. Hagemann, T. Hayakawa, T. Komatsubara, A. Maj, S. Mitarai, M. Oshima, J. Sampson, T. Shizuma, P.G. Varmette, Eur. Phys. J. A 3, 197 (1998).

18. M.M. Minor, R.K. Sheline, E.T. Jurney, Phys. Rev. C 3, 766 (1971).

19. M. Dasgupta, G.D. Dracoulis, P.M. Walker, A.P. Byrne, T. Kibédi, F.G. Kondev, G.J. Lane, P.H. Regan, Phys. Rev. C 61, 044321 (2000).

20. T. Shizuma, Y. Toh, M. Oshima, M. Sugawara, M. Matsuda, T. Hayakawa, M. Koizumi, A. Osa, Y.H. Zhang, Z. Liu, Eur. Phys. J. A 17, 159 (2003).

21. F.G. Kondev, G.D. Dracoulis, A.P. Byrne, M. Dasgupta, T. Kibédi, G.J. Lane, Nucl. Phys. A 601, 195 (1996).

22. C.J. Gallagher, S.A. Moszkowski, Phys. Rev. 111, 1282 (1958).

23. P. Kleinheinz, P.J. Daly, R.F. Casten, Nucl. Phys. A 208, 93 (1973).

24. C. Wheldon, P.M. Walker, P.H. Regan, T. Saitoh, N. Hashimoto, G. Sletten, F.R. Xu, Nucl. Phys. A 652, 103 (1999). 